\title{
CRYSTAL STRUCTURE ON RIGGED CONFIGURATIONS
}

\author{
ANNE SCHILLING
}

\begin{abstract}
Rigged configurations are combinatorial objects originating from the Bethe Ansatz, that label highest weight crystal elements. In this paper a new unrestricted set of rigged configurations is introduced for types $A D E$ by constructing a crystal structure on the set of rigged configurations. In type $A$ an explicit characterization of unrestricted rigged configurations is provided which leads to a new fermionic formula for unrestricted Kostka polynomials or $q$-supernomial coefficients. The affine crystal structure for type $A$ is obtained as well.
\end{abstract}

\section{INTRODUCTION}

There are (at least) two main approaches to solvable lattice models and their associated quantum spin chains: the Bethe Ansatz [6] and the corner transfer matrix method [5].

In his 1931 paper [6], Bethe solved the Heisenberg spin chain based on the string hypothesis which asserts that the eigenvalues of the Hamiltonian form certain strings in the complex plane as the size of the system tends to infinity. The Bethe Ansatz has been applied to many models to prove completeness of the Bethe vectors. The eigenvalues and eigenvectors of the Hamiltonian are indexed by rigged configurations. However, numerical studies indicate that the string hypothesis is not always true [3].

The corner transfer matrix (CTM) method, introduced by Baxter [5], labels the eigenvectors by one-dimensional lattice paths. These lattice paths have a natural interpretation in terms of Kashiwara's crystal base theory [18, 19], namely as highest weight crystal elements in a tensor product of finite-dimensional crystals.

Even though neither the Bethe Ansatz nor the corner transfer matrix method are mathematically rigorous, they suggest that the existence of a bijection between the two index sets, namely rigged configurations on the one hand and highest weight crystal paths on the other (see Figure 1). For the special case when the spin chain is defined on $V_{\left(\mu_{1}\right)} \otimes$ $V_{\left(\mu_{2}\right)} \otimes \cdots \otimes V_{\left(\mu_{k}\right)}$, where $V_{\left(\mu_{i}\right)}$ is the irreducible GL $(n)$ representation indexed by the partition $\left(\mu_{i}\right)$ for $\mu_{i} \in \mathbb{N}$, a bijection between rigged configurations and semi-standard Young tableaux was given by Kerov, Kirillov and Reshetikhin [27, 28]. This bijection was proven and extended to the case when the $\left(\mu_{i}\right)$ are any sequence of rectangles in [30]. The bijection has many amazing properties. For example it takes the cocharge statistics $c c$ defined on rigged configurations to the coenergy statistics $D$ defined on crystals.

Rigged configurations and crystal paths also exist for other types. In $[16,17]$ the existence of Kirillov-Reshetikhin crystals $B^{r, s}$ was conjectured, which can be naturally associated with the dominant weight $s \Lambda_{r}$ where $s$ is a positive integer and $\Lambda_{r}$ is the $r$-th fundamental weight of the underlying algebra of finite type. For a tensor product of KirillovReshetikhin crystals $B=B^{r_{k}, s_{k}} \otimes \cdots \otimes B^{r_{1}, s_{1}}$ and a dominant weight $\Lambda$ let $\overline{\mathcal{P}}(B, \Lambda)$ be the set of all highest weight elements of weight $\Lambda$ in $B$. In the same papers $[16,17]$,

Date: July 2005.

Partially supported by NSF grants DMS-0200774 and DMS-0501101. 


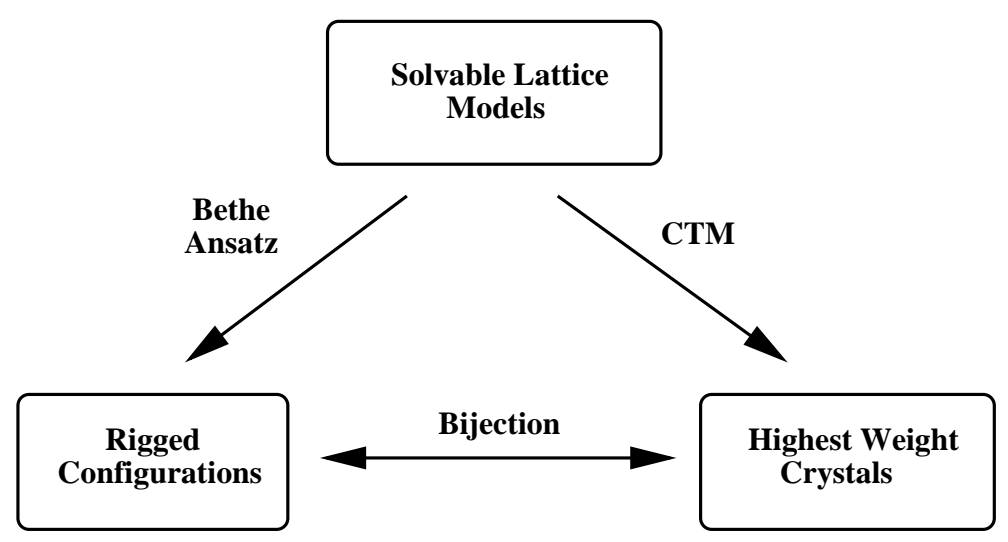

FIGURE 1. Schematic origin of rigged configurations and crystal paths

fermionic formulas $\bar{M}(L, \Lambda)$ for the one-dimensional configuration sums $\bar{X}(B, \Lambda):=$ $\sum_{b \in \overline{\mathcal{P}}(B, \Lambda)} q^{D(b)}$ were conjectured. The fermionic formulas admit a combinatorial interpretation in terms of the set of rigged configurations $\overline{\mathrm{RC}}(L, \Lambda)$, where $L$ is the multiplicity array of $B$ (see Section 3.1). A statistic preserving bijection $\Phi: \overline{\mathcal{P}}(B, \Lambda) \rightarrow \overline{\mathrm{RC}}(L, \Lambda)$ has been proven in various cases $[30,33,37,39]$ which implies the following identity

$$
\bar{X}(B, \Lambda):=\sum_{b \in \overline{\mathcal{P}}(B, \Lambda)} q^{D(b)}=\sum_{(\nu, J) \in \overline{\mathrm{RC}}(L, \Lambda)} q^{c c(\nu, J)}=: \bar{M}(L, \Lambda) .
$$

Since the sets in (1.1) are finite, these are polynomials in $q$. When $B=B^{1, s_{k}} \otimes \cdots \otimes B^{1, s_{1}}$ of type $A$, they are none other than the Kostka-Foulkes polynomials.

Rigged configurations corresponding to highest weight crystal paths are only the tip of an iceberg. In this paper we extend the definition of rigged configurations to all crystal elements in types $A D E$ by the explicit construction of a crystal structure on the set of unrestricted rigged configurations (see Definition 3.3). The proof uses Stembridge's local characterization of simply-laced crystals [41]. For nonsimply-laced algebras, the local rules provided in [41] are still necessary, but no longer sufficient conditions to characterize crystals. Crystal operators for rigged configurations associated to nonsimply-laced algebras can be constructed from the ones presented here via "folding" of the Dynkin diagrams as in the construction of virtual crystals $[34,35]$.

The equivalence of the crystal structures on rigged configurations and crystal paths together with the correspondence for highest weight vectors yields the equality of generating functions in analogy to (1.1) (see Theorem 3.10 and Corollary 3.11). Denote the unrestricted set of paths and rigged configurations by $\mathcal{P}(B, \Lambda)$ and $\mathrm{RC}(L, \Lambda)$, respectively. The corresponding generating functions $X(B, \Lambda)=M(L, \Lambda)$ are unrestricted generalized Kostka polynomials or $q$-supernomial coefficients. A direct bijection $\Phi: \mathcal{P}(B, \Lambda) \rightarrow$ $\mathrm{RC}(L, \Lambda)$ for type $A$ along the lines of [30] is constructed in $[9,10]$.

Rigged configurations are closely tied to fermionic formulas. Fermionic formulas are explicit expressions for the partition function of the underlying physical model which reflect their particle structure. For more details regarding the background of fermionic formulas see $[16,22,23]$. For type $A$ we obtain an explicit characterization of the unrestricted rigged configurations in terms of lower bounds on quantum numbers (see Definition 4.3 
and Theorem 4.6) which yields a new fermionic formula for unrestricted Kostka polynomials of type $A$ (see Equation (4.2)). Surprisingly, this formula is different from the fermionic formulas in $[15,20]$ obtained in the special cases of $B=B^{1, s_{k}} \otimes \cdots \otimes B^{1, s_{1}}$ and $B=B^{r_{k}, 1} \otimes \cdots \otimes B^{r_{1}, 1}$. The rigged configurations corresponding to the fermionic formulas of $[15,20]$ were related to ribbon tableaux and the cospin generating functions of Lascoux, Leclerc, Thibon [31,32] in reference [36]. To distinguish these rigged configurations from the ones introduced in this paper, let us call them ribbon rigged configurations.

The Lascoux-Leclerc-Thibon (LLT) polynomials [31, 32] have recently made their debut in the theory of Macdonald polynomials in the seminal paper by Haiman, Haglund, Loehr [11]. The main obstacle in obtaining a combinatorial formula for the MacdonaldKostka polynomials is the Schur positivity of certain LLT polynomials. A related problem is the conjecture of Kirillov and Shimozono [29] that the cospin generating function of ribbon tableaux equals the generalized Kostka polynomial. A possible avenue to prove this conjecture would be a direct bijection between the unrestricted rigged configurations of this paper and ribbon rigged configurations.

For type $A$ we can also describe the affine crystal operators $e_{0}$ and $f_{0}$ on rigged configurations (see Section 4.3). A level- $\ell$ restricted element $b$ in a crystal $B$ is characterized by $e_{0}^{\ell+1} b=0$. It is striking that the description of the unrestricted rigged configurations of type $A$ (see Definition 4.3) is very similar to the characterization of level-restricted rigged configurations as given in [38]. Instead of a modification of the vacancy numbers, which comprise upper bounds for the quantum numbers, an alteration of the lower bounds occurs. In both cases the modification is governed by a set of tableaux depending on the weight $\Lambda$. It would be interesting to understand the relation between the conditions imposed by classical restriction and level-restriction in a more precise manner.

One of the motivations for considering unrestricted rigged configurations was Takagi's work [42] on the inverse scattering transform, which provides a bijection between states in the $\mathfrak{s l}_{2}$ box ball system and rigged configurations. In this setting rigged configurations play the role of action-angle variables. Box ball systems can be produced from crystals of solvable lattice models for algebras other than $\mathfrak{s l}_{2}[12,13,14]$. The inverse scattering transform can be generalized to the $\mathfrak{s l}_{n}$ case [26], which should give a box-ball interpretation of the unrestricted rigged configurations presented here.

Another motivation for the study of unrestricted configuration sums, fermionic formulas and associated rigged configurations is their appearance in generalizations of the Bailey lemma [2, 43]. The Andrews-Bailey construction $[1,4]$ relies on an iterative transformation property of the $q$-binomial coefficient, which is one of the simplest unrestricted configuration sums, and can be used to prove infinite families of Rogers-Ramanujan type identities. The explicit formulas provided in this paper might trigger further progress towards generalizations to higher-rank or other types of the Andrews-Bailey construction.

The paper is organized as follows. In Section 2.1 we review basics about crystal bases and Stembridge's local characterization of crystals. In Section 3 we define rigged configurations and the new crystal structure for types $A D E$. Section 4 is devoted to type $A$, where we give an explicit characterization of the unrestricted rigged configurations in Section 4.1, a new fermionic formula for unrestricted Kostka polynomials in Section 4.2, and the affine crystal structure in Section 4.3.

Acknowledgment. I would like to thank Mark Haiman, Mark Shimozono and John Stembridge for helpful discussions, Peter Littelmann for drawing my attention to reference [41], and Masato Okado for his explanations of the box ball system [26]. I would also like to thank Lipika Deka for collaboration on $[9,10]$. 


\section{CRYSTAL GRAPHS}

We review the axiomatic definition of crystal graphs in Section 2.1 and the local characterization of crystals corresponding to representations of simply-laced algebras provided by Stembridge [41] in Section 2.2. In Section 2.3 we review the main properties of KirillovReshetikhin crystals.

2.1. Axiomatic definition. Kashiwara $[18,19]$ introduced a crystal as an edge-colored directed graph satisfying a simple set of axioms. Let $\mathfrak{g}$ be a symmetrizable Kac-Moody algebra with associated root, coroot and weight lattices $Q, Q^{\vee}, P$. Let $I$ be the index set of the Dynkin diagram and denote the simple roots, simple coroots and fundamental weights by $\alpha_{i}, h_{i}$ and $\Lambda_{i}(i \in I)$, respectively. There is a natural pairing $\langle\cdot, \cdot\rangle: Q^{\vee} \otimes P \rightarrow \mathbb{Z}$ defined by $\left\langle h_{i}, \Lambda_{j}\right\rangle=\delta_{i j}$.

The vertices of the crystal graph are elements of a set $B$. The edges of the crystal graph are colored by the index set $I$. A $P$-weighted $I$-crystal satisfies the following properties:

(1) Fix an $i \in I$. If all edges are removed except those colored $i$, the connected components are finite directed linear paths called the $i$-strings of $B$. Given $b \in B$, define $f_{i}(b)$ (resp. $e_{i}(b)$ ) to be the vertex following (resp. preceding) $b$ in its $i$ string; if there is no such vertex, declare $f_{i}(b)$ (resp. $e_{i}(b)$ ) to be undefined. Define $\varphi_{i}(b)$ (resp. $\left.\varepsilon_{i}(b)\right)$ to be the number of arrows from $b$ to the end (resp. beginning) of its $i$-string.

(2) There is a function wt $: B \rightarrow P$ such that

$$
\begin{aligned}
\operatorname{wt}\left(f_{i}(b)\right) & =\operatorname{wt}(b)-\alpha_{i} \\
\varphi_{i}(b)-\varepsilon_{i}(b) & =\left\langle h_{i}, \operatorname{wt}(b)\right\rangle .
\end{aligned}
$$

2.2. Local characterization of crystals. Kashiwara [18, 19] constructed crystal graphs for representations of $U_{q}(\mathfrak{g})$. Crystals of representations form a special subclass of the set of all crystals. In [41], Stembridge determines a simple set of local axioms that uniquely characterize the crystals corresponding to representations for simply-laced algebras.

Let $A=\left[A_{i j}\right]_{i, j \in I}$ be the Cartan matrix of a simply-laced Kac-Moody algebra $\mathfrak{g}$. Let $X$ be an edge-colored graph. Stembridge [41] introduces the notion of $A$-regularity by requiring the conditions (P1)-(P6), (P5'), (P6') to hold.

(P1) All monochromatic directed paths in $X$ have finite length. In particular $X$ has no monochromatic circuits.

(P2) For every vertex $x$ and every $i \in I$, there is at most one edge $y \stackrel{i}{\longrightarrow} x$, and dually, at most one edge $x \stackrel{i}{\longrightarrow} z$.

In the notation of the previous section, the relation $f_{i}(x)=y$ or equivalently $e_{i}(y)=x$ is graphically depicted by $x \stackrel{i}{\longrightarrow} y$. Set $\delta_{i}(x)=-\varepsilon_{i}(x)$ with $\varepsilon_{i}(x)$ and $\varphi_{i}(x)$ as defined in Section 2.1. Define

$$
\Delta_{i} \delta_{j}(x)=\delta_{j}\left(e_{i} x\right)-\delta_{j}(x), \quad \Delta_{i} \varphi_{j}(x)=\varphi_{j}\left(e_{i} x\right)-\varphi_{j}(x),
$$

whenever $e_{i} x$ is defined, and

$$
\nabla_{i} \delta_{j}(x)=\delta_{j}(x)-\delta_{j}\left(f_{i} x\right), \quad \nabla_{i} \varphi_{j}(x)=\varphi_{j}(x)-\varphi_{j}\left(f_{i} x\right)
$$

whenever $f_{i} x$ is defined.

For fixed $x \in X$ and a distinct pair $i, j \in I$, assuming that $e_{i} x$ is defined, require

(P3) $\Delta_{i} \delta_{j}(x)+\Delta_{i} \varphi_{j}(x)=A_{i j}$, and

(P4) $\Delta_{i} \delta_{j}(x) \leq 0, \Delta_{i} \varphi_{j}(x) \leq 0$. 
Note that for simply-laced algebras $A_{i j} \in\{0,-1\}$ for $i, j \in I$ distinct. Hence (P3) and (P4) allow for only three possibilities:

$$
\left(A_{i j}, \Delta_{i} \delta_{j}(x), \Delta_{i} \varphi_{j}(x)\right)=(0,0,0),(-1,-1,0),(-1,0,-1) .
$$

Assuming that $e_{i} x$ and $e_{j} x$ both exist, we require

(P5) $\Delta_{i} \delta_{j}(x)=0$ implies $y:=e_{i} e_{j} x=e_{j} e_{i} x$ and $\nabla_{j} \varphi_{i}(y)=0$.

(P6) $\Delta_{i} \delta_{j}(x)=\Delta_{j} \delta_{i}(x)=-1$ implies $y:=e_{i} e_{j}^{2} e_{i} x=e_{j} e_{i}^{2} e_{j} x$ and $\nabla_{i} \varphi_{j}(y)=$ $\nabla_{j} \varphi_{i}(y)=-1$.

Dually, assuming that $f_{i} x$ and $f_{j} x$ both exist, we require

(P5') $\nabla_{i} \varphi_{j}(x)=0$ implies $y:=f_{i} f_{j} x=f_{j} f_{i} x$ and $\Delta_{j} \delta_{i}(y)=0$.

(P6') $\nabla_{i} \varphi_{j}=\nabla_{j} \varphi_{i}(x)=-1$ implies $y:=f_{i} f_{j}^{2} f_{i} x=f_{j} f_{i}^{2} f_{j} x$ and $\Delta_{i} \delta_{j}(y)=$ $\Delta_{j} \delta_{i}(y)=-1$.

Definition 2.1. [41, Definition 1.1] Let $A$ be a simply-laced Cartan matrix. An edgecolored directed graph is $A$-regular if it satisfies (P1)-(P6) and (P5')-(P6').

Stembridge proved [41, Proposition 1.4] that any two $A$-regular posets $P, P^{\prime}$ with maximal elements $x, x^{\prime}$ are isomorphic if and only if $\varphi_{i}(x)=\varphi_{i}\left(x^{\prime}\right)$ for all $i \in I$. Moreover this isomorphism is unique. Let $\Lambda=\sum_{i \in I} \mu_{i} \Lambda_{i}$ be a dominant weight. Denote by $B(\Lambda)$ the unique $A$-regular poset with maximal element $b$ such that $\varphi_{i}(b)=\mu_{i}$ for all $i \in I$.

Theorem 2.2. [41, Theorem 3.3] If $A$ is a simply-laced Cartan matrix, then the crystal graph of the irreducible $U_{q}(A)$-module of highest weight $\Lambda$ is $B(\Lambda)$.

2.3. Kirillov-Reshetikhin crystals. Kirillov-Reshetikhin crystals are crystals for finitedimensional irreducible modules over quantum affine algebras. The irreducible finitedimensional $U_{q}^{\prime}(\mathfrak{g})$-modules were classified by Chari and Pressley $[7,8]$ in terms of Drinfeld polynomials. Here $U_{q}^{\prime}(\mathfrak{g})$ is the derived algebra without the generator $q^{d}$, where $d$ is degree operator in $\mathfrak{g}$. The Kirillov-Reshetikhin modules $W^{r, s}$, labeled by a Dynkin node $r$ of the underlying algebra of finite type and a positive integer $s$, form a special class of these finite-dimensional modules. They naturally correspond to the weight $s \Lambda_{r}$, where $\Lambda_{r}$ is the $r$-th fundamental weight of $\mathfrak{g}$. It was conjectured in $[16,17]$, that there exists a crystal $B^{r, s}$ for each $W^{r, s}$. In general, the existence of $B^{r, s}$ is still an open question. For type $A_{n}^{(1)}$ the crystal $B^{r, s}$ is known to exist [25] and its combinatorial structure has been studied [40]. For other types, the existence and combinatorial structure of $B^{r, s}$ has been considered in various articles (see for example [21, 24, 25]). As classical crystals the Kirillov-Reshetikhin crystals are isomorphic to

$$
B^{r, s} \simeq B\left(s \Lambda_{r}\right) \oplus \bigoplus_{\Lambda} B(\Lambda)
$$

where $B(\Lambda)$ is the classically highest weight crystal of highest weight $\Lambda$ and the sum is over a particular set of weights contained in $s \Lambda_{r}$ (for more details see [16]).

\section{CRystal Structure on RigGed CONFigurations}

In this section we define a crystal structure on rigged configurations. As alluded to in the introduction rigged configurations form a combinatorial set to index the eigenvalues and eigenvectors of the Hamiltonian of an exactly solvable lattice model. The simplest version of rigged configurations appeared in Bethe's original paper [6] and was later generalized by Kerov, Kirillov and Reshetikhin [27, 28] to models with GL $(n)$ symmetry. Since the eigenvectors of the Hamiltonian can also be viewed as highest weight vectors, 
one expects a bijection between rigged configurations and semi-standard Young tableaux in the GL $(n)$ case. Such a bijection was given in [28, 30]. Rigged configurations for other types follow from the fermionic formulas given in $[16,17]$ and they correspond to highest weight crystal elements [33, 37, 39]. Here we extend the notion of rigged configurations to non-highest weight elements called unrestricted rigged configurations and define a crystal structure on this set. In Section 3.1 we review the definition and known results about the usual rigged configurations. In Section 3.2 the set of unrestricted rigged configurations is introduced and the crystal structure is defined for types $A D E$ (Definition 3.3). This leads to a bijection between crystal paths and unrestricted rigged configurations (Theorem 3.10) and the equality of generating functions (Corollary 3.11).

3.1. Definition of rigged configurations. Let $\mathfrak{g}$ be a simple simply-laced affine KacMoody algebra. Define $\bar{I}=I \backslash\{0\}$ the index set of the underlying algebra of finite type and set $\mathcal{H}=\bar{I} \times \mathbb{Z}_{>0}$. The (highest-weight) rigged configurations are indexed by a multiplicity array $L=\left(L_{i}^{(a)} \mid(a, i) \in \mathcal{H}\right)$ of nonnegative integers and a dominant weight $\Lambda$. The sequence of partitions $\nu=\left\{\nu^{(a)} \mid a \in \bar{I}\right\}$ is a $(L, \Lambda)$-configuration if

$$
\sum_{(a, i) \in \mathcal{H}} i m_{i}^{(a)} \alpha_{a}=\sum_{(a, i) \in \mathcal{H}} i L_{i}^{(a)} \Lambda_{a}-\Lambda
$$

where $m_{i}^{(a)}$ is the number of parts of length $i$ in partition $\nu^{(a)}$. Denote the set of all $(L, \Lambda)$ configurations by $\mathrm{C}(L, \Lambda)$. The vacancy number of a configuration is defined as

$$
p_{i}^{(a)}=\sum_{j \geq 1} \min (i, j) L_{j}^{(a)}-\sum_{(b, j) \in \mathcal{H}}\left(\alpha_{a} \mid \alpha_{b}\right) \min (i, j) m_{j}^{(b)} .
$$

Here $(\cdot \mid \cdot)$ is the normalized invariant form on the weight lattice $P$ such that $A_{a b}=\left(\alpha_{a} \mid \alpha_{b}\right)$ is the Cartan matrix. The $(L, \Lambda)$-configuration $\nu$ is admissible if $p_{i}^{(a)} \geq 0$ for all $(a, i) \in$ $\mathcal{H}$, and the set of admissible $(L, \Lambda)$-configurations is denoted by $\overline{\mathrm{C}}(L, \Lambda)$.

A rigged configuration is an admissible configuration together with a set of labels of quantum numbers. A partition can be viewed as a multiset of positive integers. A rigged partition is by definition a finite multiset of pairs $(i, x)$ where $i$ is a positive integer and $x$ is a nonnegative integer. The pairs $(i, x)$ are referred to as strings; $i$ is referred to as the length or size of the string and $x$ as the label or quantum number of the string. A rigged partition is said to be a rigging of the partition $\rho$ if the multiset, consisting of the sizes of the strings, is the partition $\rho$. So a rigging of $\rho$ is a labeling of the parts of $\rho$ by nonnegative integers, where one identifies labelings that differ only by permuting labels among equal sized parts of $\rho$.

A rigging $J$ of the $(L, \Lambda)$-configuration $\nu$ is a sequence of riggings of the partitions $\nu^{(a)}$ such that every label $x$ of a part of $\nu^{(a)}$ of size $i$ satisfies the inequality

$$
0 \leq x \leq p_{i}^{(a)} \text {. }
$$

Alternatively, a rigging of a configuration $\nu$ may be viewed as a double-sequence of partitions $J=\left(J^{(a, i)} \mid(a, i) \in \mathcal{H}\right)$ where $J^{(a, i)}$ is a partition that has at most $m_{i}^{(a)}$ parts each not exceeding $p_{i}^{(a)}$. The pair $(\nu, J)$ is called a rigged configuration. The set of riggings of admissible $(L, \Lambda)$-configurations is denoted by $\overline{\mathrm{RC}}(L, \Lambda)$. Let $(\nu, J)^{(a)}$ be the $a$-th rigged partition of $(\nu, J)$. The colabel or coquantum number of a string $(i, x)$ in $(\nu, J)^{(a)}$ is defined to be $p_{i}^{(a)}-x$. A string $(i, x) \in(\nu, J)^{(a)}$ is said to be singular if $x=p_{i}^{(a)}$, that is, its label takes on the maximum value. 
Using (3.2), one may easily verify that

$$
-p_{i-1}^{(a)}+2 p_{i}^{(a)}-p_{i+1}^{(a)} \geq m_{i}^{(a-1)}-2 m_{i}^{(a)}+m_{i}^{(a+1)} .
$$

This implies in particular the convexity condition

$$
p_{i}^{(a)} \geq \frac{1}{2}\left(p_{i-1}^{(a)}+p_{i+1}^{(a)}\right) \quad \text { if } m_{i}^{(a)}=0 .
$$

The set of rigged configurations is endowed with a natural statistic $c c$ called cocharge. For a configuration $\nu \in \overline{\mathrm{C}}(L, \Lambda)$ define

$$
c c(\nu)=\frac{1}{2} \sum_{(a, j),(b, k) \in \mathcal{H}}\left(\alpha_{a} \mid \alpha_{b}\right) \min (j, k) m_{j}^{(a)} m_{k}^{(b)} .
$$

For a rigged configuration $(\nu, J) \in \overline{\mathrm{RC}}(L, \Lambda)$ set

$$
c c(\nu, J)=c c(\nu)+\sum_{(a, i) \in \mathcal{H}}\left|J^{(a, i)}\right|,
$$

where $\left|J^{(a, i)}\right|$ is the size of partition $J^{(a, i)}$.

As mentioned in the introduction, rigged configurations correspond to highest weight crystal elements. Let $B^{r, s}$ be a Kirillov-Reshetikhin crystal for $(r, s) \in \mathcal{H}$ and $B=$ $B^{r_{k}, s_{k}} \otimes B^{r_{k-1}, s_{k-1}} \otimes \cdots \otimes B^{r_{1}, s_{1}}$. Associate to $B$ the multiplicity array $L=\left(L_{s}^{(r)}\right.$ $(r, s) \in \mathcal{H})$ where $L_{s}^{(r)}$ counts the number of tensor factors $B^{r, s}$ in $B$. Denote by

$$
\overline{\mathcal{P}}(B, \Lambda)=\left\{b \in B \mid \operatorname{wt}(b)=\Lambda, e_{i}(b) \text { undefined for all } i \in \bar{I}\right\}
$$

the set of all highest weight elements of weight $\Lambda$ in $B$. There is a natural statistics defined on $B$, called energy function or more precisely tail coenergy function $D: B \rightarrow \mathbb{Z}$ (see [39, Eq. (5.1)] for a precise definition).

The following theorem was proven in [30] for type $A_{n-1}^{(1)}$ and general $B=B^{r_{k}, s_{k}} \otimes$ $\cdots \otimes B^{r_{1}, s_{1}}$, in [37] for type $D_{n}^{(1)}$ and $B=B^{r_{k}, 1} \otimes \cdots \otimes B^{r_{1}, 1}$ and in [39] for type $D_{n}^{(1)}$ and $B=B^{1, s_{k}} \otimes \cdots \otimes B^{1, s_{1}}$.

Theorem 3.1. [30, 37, 39] For $\Lambda$ a dominant weight, $B$ as above and $L$ the corresponding multiplicity array, there is a bijection $\bar{\Phi}: \overline{\mathcal{P}}(B, \Lambda) \rightarrow \overline{\mathrm{RC}}(L, \Lambda)$ which preserves the statistics, that is, $D(b)=c c(\bar{\Phi}(b))$ for all $b \in \overline{\mathcal{P}}(B, \Lambda)$.

Defining the generating functions

$$
\begin{aligned}
& \bar{X}(B, \Lambda)=\sum_{b \in \overline{\mathcal{P}}(B, \Lambda)} q^{D(b)}, \\
& \bar{M}(L, \Lambda)=\sum_{(\nu, J) \in \overline{\operatorname{RC}}(L, \Lambda)} q^{c c(\nu, J)},
\end{aligned}
$$

we get the immediate corollary of Theorem 3.1.

Corollary 3.2. [30, 37, 39] Let $\Lambda, B$ and L as in Theorem 3.1. Then $\bar{X}(B, \Lambda)=\bar{M}(L, \Lambda)$.

3.2. Crystal structure. In this section we introduce the set of unrestricted rigged configurations $\mathrm{RC}(L)$ by defining a crystal structure generated from highest weight vectors given by elements in $\overline{\mathrm{RC}}(L)=\bigcup_{\Lambda \in P^{+}} \overline{\mathrm{RC}}(L, \Lambda)$ by the Kashiwara operators $e_{a}, f_{a}$.

Definition 3.3. Let $L$ be a multiplicity array. Define the set of unrestricted rigged configurations $\mathrm{RC}(L)$ as the set generated from the elements in $\overline{\mathrm{RC}}(L)$ by the application of the operators $f_{a}, e_{a}$ for $a \in \bar{I}$ defined as follows: 
(1) Define $e_{a}(\nu, J)$ by removing a box from a string of length $k$ in $(\nu, J)^{(a)}$ leaving all colabels fixed and increasing the new label by one. Here $k$ is the length of the string with the smallest negative rigging of smallest length. If no such string exists, $e_{a}(\nu, J)$ is undefined.

(2) Define $f_{a}(\nu, J)$ by adding a box to a string of length $k$ in $(\nu, J)^{(a)}$ leaving all colabels fixed and decreasing the new label by one. Here $k$ is the length of the string with the smallest nonpositive rigging of largest length. If no such string exists, add a new string of length one and label -1 . If the result is not a valid unrestricted rigged configuration $f_{a}(\nu, J)$ is undefined.

Let $(\nu, J) \in \operatorname{RC}(L)$. If $f_{a}$ adds a box to a string of length $k$ in $(\nu, J)^{(a)}$, then the vacancy numbers change according to

$$
p_{i}^{(b)} \mapsto p_{i}^{(b)}-\left(\alpha_{a} \mid \alpha_{b}\right) \chi(i>k),
$$

where $\chi(S)=1$ if the statement $S$ is true and $\chi(S)=0$ if $S$ is false. Similarly, if $e_{a}$ adds a box of length $k$ to $(\nu, J)^{(a)}$, then the vacancy numbers change as

$$
p_{i}^{(b)} \mapsto p_{i}^{(b)}+\left(\alpha_{a} \mid \alpha_{b}\right) \chi(i \geq k)
$$

Hence if $\left(\nu^{\prime}, J^{\prime}\right)=f_{a}(\nu, J)$ exists, it is easy to check that $e_{a}\left(\nu^{\prime}, J^{\prime}\right)=(\nu, J)$ and vice versa.

Remark 3.4. Note that it follows from (3.7) that for a string $(i, m)$ in $(\nu, J) \in \mathrm{RC}(L)$ we have $m \geq-i$. Hence, $e_{a}$ only removes a string of length 1 if its label is -1 , which in Definition 3.3 is interpreted as increasing the label by one.

We may define a weight function wt $: \mathrm{RC}(L) \rightarrow P$ as

$$
\operatorname{wt}(\nu, J)=\sum_{(a, i) \in \mathcal{H}} i\left(L_{i}^{(a)} \Lambda_{a}-m_{i}^{(a)} \alpha_{a}\right)
$$

for $(\nu, J) \in \mathrm{RC}(L)$. It is clear from the definition that $\operatorname{wt}\left(f_{a}(\nu, J)\right)=\operatorname{wt}(\nu, J)-\alpha_{a}$. Define

$$
\mathrm{RC}(L, \Lambda)=\{(\nu, J) \in \mathrm{RC}(L) \mid \operatorname{wt}(\nu, J)=\Lambda\}
$$

Example 3.5. Let $\mathfrak{g}$ be of type $A_{2}^{(1)}$. Let $\Lambda=\Lambda_{1}-\Lambda_{2}, L_{1}^{(1)}=L_{3}^{(1)}=L_{2}^{(2)}=1$ and all other $L_{i}^{(a)}=0$. Then

$$
(\nu, J)=\begin{array}{|l|l|l|l|}
\square & -1 & \square & \square \\
\hline \square & -1
\end{array}
$$

is in $\mathrm{RC}(L, \Lambda)$, where the parts of the rigging $J^{(a, i)}$ are written next to the parts of length $i$ in partition $\nu^{(a)}$. We have

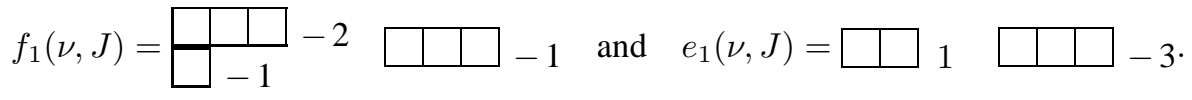

Before stating our main result, we need some preliminary properties of the crystal operators.

Lemma 3.6. Let $(\nu, J) \in \mathrm{RC}(L)$. For fixed $a \in \bar{I}$, let $p=p_{i}^{(a)}$ be the vacancy number for large $i$ and let $s \leq 0$ be the smallest nonpositive label in $(\nu, J)^{(a)}$; if no such label exists set $s=0$. Then $\varphi_{a}(\nu, J)=p-s$. 
Proof. By definition, $f_{a}$ adds a box to the largest string with $s$-rigging in $(\nu, J)^{(a)}$. Let the length of this string be $k_{0}$. By the maximality of $k_{0}$, we have $p_{j}^{(a)}>s$ for all $j>k_{0}$ such that $j$ exists as a part in $\nu^{(a)}$. If $p=s$, then by the convexity (3.4) of $p_{j}^{(a)}$ we have $m_{j}^{(a)}=0$ for $j>k_{0}$ and $p_{k_{0}+1}^{(a)}=s$. Under the application of $f_{a}$, the new label of the string of length $k_{0}+1$ would be $s-1$, but the vacancy number changes by -2 . Hence $\varphi_{a}(\nu, J)=0=p-s$ in this case. If $p>s$, by the convexity (3.4) of $p_{i}^{(a)}$ we have that $p_{j}^{(a)}>s$ for all $j>k_{0}$ and $f_{a}(\nu, J)$ is defined. The new label is $s-1$ and $p_{j}^{(a)} \mapsto p_{j}^{(a)}-2 \geq s-1$ for all $j>k_{0}$. By induction on $m \geq 1, f_{a}$ adds a box to the largest string with rigging $s-m$ of $f_{a}^{m}(\nu, J)^{(a)}$. Let $k_{m}>k_{m-1}$ be the length of this string. For large $i$, the vacancy number is $p-2 m$. Suppose that $p>s+m$. Again by the maximality of $k_{m}$ and the convexity of the vacancy numbers (3.4), the vacancy numbers of $f_{a}^{m}(\nu, J)$ satisfy $p_{j}^{(a)}>s-m$ for all $j>k_{m}$. The new label of the added box is $s-m-1$ and compared to the original vacancy number $p_{j}^{(a)} \mapsto p_{j}^{(a)}-2(m+1) \geq s-m-1$ for all $j>k_{m}$. If $p=s+m$, then the new label becomes $s-m-1$, but the vacancy number is $p-2 m-2=s-m-2$. Hence $f_{a}^{m+1}(\nu, J)$ is not defined in this case. This proves $\varphi_{a}(\nu, J)=p-s$.

Theorem 3.7. Let $\mathfrak{g}$ be of simply-laced type. For $(\bar{\nu}, \bar{J}) \in \overline{\mathrm{RC}}(L, \Lambda)$, let $X_{(\bar{\nu}, \bar{J})}$ be the graph generated by $(\bar{\nu}, \bar{J})$ and $e_{a}, f_{a}$ for $a \in \bar{I}$. Then $X_{(\bar{\nu}, \bar{J})}$ is isomorphic to the crystal graph $B(\Lambda)$.

Proof. Let $A=\left[A_{a b}\right]$ be a Cartan matrix of simply-laced type and $\Lambda=\sum_{a \in \bar{I}} \mu_{a} \Lambda_{a}$. By Theorem 2.2 it suffices to check that the graph $X_{(\bar{\nu}, \bar{J})}$ generated by the maximal element $(\bar{\nu}, \bar{J})$ and operators $e_{a}, f_{a}$ as defined in Definition 3.3 is $A$-regular and that $\varphi_{a}(\bar{\nu}, \bar{J})=\mu_{a}$ for all $a \in \bar{I}$.

The claim that $\varphi_{a}(\bar{\nu}, \bar{J})=\mu_{a}$ for all $a \in \bar{I}$ follows from Lemma 3.6. Combining (3.2) and (3.1) we find that $p_{i}^{(a)}=\mu_{a}$ for large $i$. Note that since $(\bar{\nu}, \bar{J}) \in \overline{\operatorname{RC}}(L, \Lambda)$, it does not have any negative riggings, so that $s=0$. Hence by Lemma 3.6, $\varphi_{a}(\bar{\nu}, \bar{J})=\mu_{a}$.

Next we check that $X_{(\bar{\nu}, \bar{J})}$ is $A$-regular. Let $(\nu, J) \in X_{(\bar{\nu}, \bar{J})}$. By Lemma (3.6), $\varphi_{a}(\nu, J)$ is finite. This proves (P1). (P2) is clear from Definition 3.3.

To prove (P3) and (P4) we show that one of the following conditions hold

$$
\left(A_{a b}, \Delta_{a} \delta_{b}(\nu, J), \Delta_{a} \varphi_{b}(\nu, J)\right)=(0,0,0),(-1,-1,0),(-1,0,-1) .
$$

It is clear from the definitions that the operators $\left[f_{a}, f_{b}\right]=\left[e_{a}, e_{b}\right]=0$ commute when $A_{a b}=0$, so that $\Delta_{a} \delta_{b}(\nu, J)=\Delta_{a} \varphi_{b}(\nu, J)=0$ in this case. Hence assume that $A_{a b}=$ -1 . Let $k_{a}^{e}$ be the length of the string in $(\nu, J)^{(a)}$ selected by $e_{a}$. Let $k_{b}^{f}$ be the length of the string in $(\nu, J)^{(b)}$ selected by $f_{b}$. Under $e_{a}$ the vacancy number changes according to

$$
p_{i}^{(b)} \mapsto p_{i}^{(b)}-\chi\left(i \geq k_{a}^{e}\right) .
$$

Therefore by Lemma (3.6) we have

$$
\Delta_{a} \varphi_{b}(\nu, J)= \begin{cases}0 & \text { if } k_{a}^{e} \leq k_{b}^{f}, \\ -1 & \text { if } k_{a}^{e}>k_{b}^{f} .\end{cases}
$$

Similarly, it follows from (3.9) that

$$
\Delta_{a} \delta_{b}(\nu, J)= \begin{cases}-1 & \text { if } k_{a}^{e} \leq k_{b}^{f}, \\ 0 & \text { if } k_{a}^{e}>k_{b}^{f} .\end{cases}
$$


(Note that by Remark 3.4 the labels $s$ of strings of length $i$ in $(\nu, J)^{(b)}$ satisfy $s \geq-i$ for all $(\nu, J) \in X_{(\bar{\nu}, \bar{J})}$. Hence $e_{a}(\nu, J)$ does not exist if this condition does not hold for $e_{a}(\nu, J)$ ). This proves (P3) and (P4).

For (P5) assume that $\Delta_{a} \delta_{b}(\nu, J)=0$. As before, if $A_{a b}=0$, we have $\left[e_{a}, e_{b}\right]=$ $\left[f_{a}, f_{b}\right]=0$ and $\nabla_{b} \varphi_{a}\left(\nu^{\prime}, J^{\prime}\right)=0$ for any $\left(\nu^{\prime}, J^{\prime}\right)$, hence in particular for $\left(\nu^{\prime}, J^{\prime}\right)=$ $e_{a} e_{b}(\nu, J)$. Therefore assume that $A_{a b}=-1$. By (3.11), $\Delta_{a} \delta_{b}(\nu, J)=0$ implies $k_{a}^{e}>$ $k_{b}^{f}$. An explicit calculation yields that $\left(\nu^{\prime}, J^{\prime}\right)=e_{a} e_{b}(\nu, J)=e_{b} e_{a}(\nu, J)$. Note that

$$
\begin{aligned}
\nabla_{b} \varphi_{a}\left(\nu^{\prime}, J^{\prime}\right) & =\varphi_{a}\left(e_{a} e_{b}(\nu, J)\right)-\varphi_{a}\left(f_{b} e_{b} e_{a}(\nu, J)\right) \\
& =\varphi_{a}\left(e_{b}(\nu, J)\right)+1-\varphi_{a}(\nu, J)-1=\Delta_{b} \varphi_{a}(\nu, J) .
\end{aligned}
$$

Since $k_{b}^{e} \leq k_{b}^{f}<k_{a}^{e} \leq k_{a}^{f}$ it follows from (3.10) that $\nabla_{b} \varphi_{a}\left(\nu^{\prime}, J^{\prime}\right)=\Delta_{b} \varphi_{a}(\nu, J)=0$.

For (P6) assume that $\Delta_{a} \delta_{b}(\nu, J)=\Delta_{b} \delta_{a}(\nu, J)=-1$. In this case $k_{a}^{e} \leq k_{b}^{f}$ and $k_{b}^{e} \leq k_{a}^{f}$, and by definition $k_{a}^{e} \leq k_{a}^{f}, k_{b}^{e} \leq k_{b}^{f}$. It can be checked explicitly that $\left(\nu^{\prime}, J^{\prime}\right):=$ $e_{a} e_{b}^{2} e_{a}(\nu, J)=e_{b} e_{a}^{2} e_{b}(\nu, J)$ in this case. Also,

$$
\begin{aligned}
& \nabla_{a} \varphi_{b}\left(\nu^{\prime}, J^{\prime}\right)=\varphi_{b}\left(e_{b} e_{a}^{2} e_{b}(\nu, J)\right)-\varphi_{b}\left(f_{a} e_{a} e_{b}^{2} e_{a}(\nu, J)\right) \\
& \quad=\varphi_{b}\left(e_{a}^{2} e_{b}(\nu, J)\right)+1-\varphi_{b}\left(e_{a}(\nu, J)\right)-2=\varphi_{b}\left(e_{a}^{2} e_{b}(\nu, J)\right)-\varphi_{b}\left(e_{a}(\nu, J)\right)-1 .
\end{aligned}
$$

It can be shown explicitly that $\varphi_{b}\left(e_{a}^{2} e_{b}(\nu, J)\right)=\varphi_{b}\left(e_{a}(\nu, J)\right)=\varphi_{b}(\nu, J)$, which implies that $\nabla_{a} \varphi_{b}\left(\nu^{\prime}, J^{\prime}\right)=-1$ and similarly with $a$ and $b$ interchanged.

$\left(\mathrm{P}^{\prime}\right)$ and (P6') can be proved analogously.

Example 3.8. Consider the crystal $B\left(\Lambda_{1}+\Lambda_{2}\right)$ of type $A_{2}$ in $B=\left(B^{1,1}\right)^{\otimes 3}$. Here is the crystal graph in the usual labeling and the rigged configuration labeling:
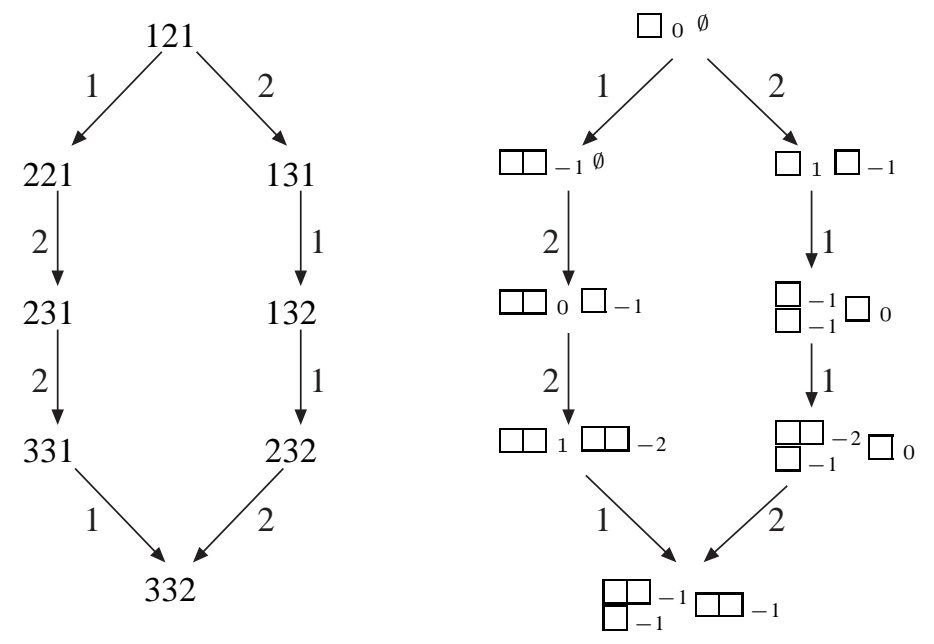

Theorem 3.9. Let $X_{(\bar{\nu}, \bar{J})}$ be as in Theorem 3.7. The cocharge cc as defined in (3.5) is constant on $X_{(\bar{\nu}, \bar{J})}$.

Proof. Let $(\nu, J) \in X_{(\bar{\nu}, \bar{J})}$ such that $f_{a}(\nu, J)$ is defined. It is easy to check that adding a box to a string of length $k$ in $(\nu, J)^{(a)}$ changes the cocharge by

$$
c c(\nu) \mapsto c c(\nu)+1+\sum_{b, i}\left(\alpha_{a} \mid \alpha_{b}\right) \chi(i>k) m_{i}^{(b)} .
$$


Since $f_{a}$ changes the label of the new string by -1 and leaves the colabels of all other strings unchanged, it is clear comparing with (3.7) that $f_{a}$ does not change the total cocharge, that is $c c(\nu, J)=c c\left(f_{a}(\nu, J)\right)$.

For $B=B^{r_{k}, s_{k}} \otimes \cdots \otimes B^{r_{1}, s_{1}}$ and $\Lambda \in P$ let

$$
\mathcal{P}(B, \Lambda)=\{b \in B \mid \operatorname{wt}(b)=\Lambda\} .
$$

Theorem 3.10. Let $\Lambda \in P, B$ be as in Theorem 3.1 and $L$ the corresponding multiplicity array. Then there is a bijection $\Phi: \mathcal{P}(B, \Lambda) \rightarrow \mathrm{RC}(L, \Lambda)$ which preserves the statistics, that is, $D(b)=c c(\Phi(b))$ for all $b \in \mathcal{P}(B, \Lambda)$.

Proof. By Theorem 3.1 there is such a bijection for the maximal elements $b \in \overline{\mathcal{P}}(B)$. By Theorems 3.7 and 3.9 this extends to all of $\mathcal{P}(B, \Lambda)$.

Extending the definitions of (3.6) to

$$
\begin{aligned}
& X(B, \Lambda)=\sum_{b \in \mathcal{P}(B, \Lambda)} q^{D(b)}, \\
& M(L, \Lambda)=\sum_{(\nu, J) \in \operatorname{RC}(L, \Lambda)} q^{c c(\nu, J)},
\end{aligned}
$$

we obtain the corollary:

Corollary 3.11. With all hypotheses of Theorem 3.10, we have $X(B, \Lambda)=M(L, \Lambda)$.

\section{UNRESTRICTED RIGGED CONFIGURATIONS FOR TYPE $A_{n-1}^{(1)}$}

In this section we give an explicit description of the elements in $\mathrm{RC}(L, \Lambda)$ for type $A_{n-1}^{(1)}$. Generally speaking, the elements are rigged configurations where the labels lie between the vacancy number and certain lower bounds defined explicitly (Definition 4.3 and Theorem 4.6). We use this in Section 4.2 to write down an explicit fermionic formula for the unrestricted configuration sum $X(B, \Lambda)$. Section 4.3 is devoted to the affine crystal structure of $\mathrm{RC}(L, \Lambda)$.

4.1. Characterization of unrestricted rigged configurations. Let $L=\left(L_{i}^{(a)} \mid(a, i) \in\right.$ $\mathcal{H})$ be a multiplicity array and $\Lambda \in P$. Recall that the set of $(L, \Lambda)$-configurations $\mathrm{C}(L, \Lambda)$ is the set of all sequences of partitions $\nu=\left(\nu^{(a)} \mid a \in \bar{I}\right)$ such that (3.1) holds. As discussed in Section 3.1, in the usual setting a rigged configuration $(\nu, J) \in \overline{\mathrm{RC}}(L, \Lambda)$ consists of a configuration $\nu \in \mathrm{C}(L, \Lambda)$ together with a double sequence of partitions $J=\left\{J^{(a, i)} \mid(a, i) \in \mathcal{H}\right\}$ such that the partition $J^{(a, i)}$ is contained in a $m_{i}^{(a)} \times p_{i}^{(a)}$ rectangle. In particular this requires that $p_{i}^{(a)} \geq 0$. The unrestricted rigged configurations $(\nu, J) \in \mathrm{RC}(L, \Lambda)$ can contain labels that are negative, that is, the lower bound on the parts in $J^{(a, i)}$ can be less than zero.

To define the lower bounds we need the following notation. Let $\lambda=\left(\lambda_{1}, \ldots, \lambda_{n}\right)$ be the $n$-tuple of nonnegative integers corresponding to $\Lambda$, that is $\Lambda=\sum_{i \in \bar{I}}\left(\lambda_{i}-\lambda_{i+1}\right) \Lambda_{i}$. In this section we use $\Lambda$ and $\lambda$ interchangeably. Let $\lambda^{\prime}=\left(c_{1}, c_{2}, \ldots, c_{n-1}\right)^{t}$, where $c_{k}=\lambda_{k+1}+\lambda_{k+2}+\cdots+\lambda_{n}$ is the length of the $k$-th column of $\lambda^{\prime}$, and let $\mathcal{A}\left(\lambda^{\prime}\right)$ be the set of tableaux of shape $\lambda^{\prime}$ such that the entries are strictly decreasing along columns, and the letters in column $k$ are from the set $\left\{1,2, \ldots, c_{k-1}\right\}$ with $c_{0}=c_{1}$. 
Example 4.1. For $n=4$ and $\lambda=(0,1,1,1)$, the set $\mathcal{A}\left(\lambda^{\prime}\right)$ consists of the following tableaux

\begin{tabular}{|c|c|c|c|c|c|}
\hline \begin{tabular}{|l|l|l|}
3 & 3 & 2 \\
\end{tabular} & \begin{tabular}{|l|l|l|}
3 & 3 & 2 \\
\end{tabular} & \begin{tabular}{|l|l|l|}
3 & 2 & 2 \\
\end{tabular} & \begin{tabular}{|l|l|l|}
3 & 3 & 1 \\
\end{tabular} & \begin{tabular}{|l|l|l|}
3 & 3 & 1 \\
\end{tabular} & \begin{tabular}{|l|l|l|}
3 & 2 & 1 \\
\end{tabular} \\
\hline \begin{tabular}{|l|l|}
2 & 2 \\
\end{tabular} & \begin{tabular}{|l|l|}
2 & 1 \\
\end{tabular} & \begin{tabular}{|l|l|}
2 & 1 \\
\end{tabular} & \begin{tabular}{|l|l|}
2 & 2 \\
\end{tabular} & \begin{tabular}{|l|l|}
2 & 1 \\
\end{tabular} & \begin{tabular}{|l|l|}
2 & 1 \\
\end{tabular} \\
\hline 1 & 1 & 1 & 1 & 1 & 1 \\
\hline
\end{tabular}

Remark 4.2. Denote by $t_{j, k}$ the entry of $t \in \mathcal{A}\left(\lambda^{\prime}\right)$ in row $j$ and column $k$. Note that $c_{k}-j+1 \leq t_{j, k} \leq c_{k-1}-j+1$ since the entries in column $k$ are strictly decreasing and lie in the set $\left\{1,2, \ldots, c_{k-1}\right\}$. This implies $t_{j, k} \leq c_{k-1}-j+1 \leq t_{j, k-1}$, so that the rows of $t$ are weakly decreasing.

Given $t \in \mathcal{A}\left(\lambda^{\prime}\right)$, we define the lower bound as

$$
M_{i}^{(a)}(t)=-\sum_{j=1}^{c_{a}} \chi\left(i \geq t_{j, a}\right)+\sum_{j=1}^{c_{a+1}} \chi\left(i \geq t_{j, a+1}\right),
$$

where recall that $\chi(S)=1$ if the the statement $S$ is true and $\chi(S)=0$ otherwise.

Let $M, p, m \in \mathbb{Z}$ such that $m \geq 0$. A $(M, p, m)$-quasipartition $\mu$ is a tuple of integers $\mu=\left(\mu_{1}, \mu_{2}, \ldots, \mu_{m}\right)$ such that $M \leq \mu_{m} \leq \mu_{m-1} \leq \cdots \leq \mu_{1} \leq p$. Each $\mu_{i}$ is called a part of $\mu$. Note that for $M=0$ this would be a partition with at most $m$ parts each not exceeding $p$.

Definition 4.3. An extended rigged configuration $(\nu, J)$ is a configuration $\nu \in \mathrm{C}(L, \lambda)$ together with a sequence $J=\left\{J^{(a, i)} \mid(a, i) \in \mathcal{H}\right\}$ where $J^{(a, i)}$ is a $\left(M_{i}^{(a)}(t), p_{i}^{(a)}, m_{i}^{(a)}\right)$ quasipartition for some $t \in \mathcal{A}\left(\lambda^{\prime}\right)$. Denote the set of all extended rigged configurations corresponding to $(L, \lambda)$ by $\widetilde{\mathrm{RC}}(L, \lambda)$.

Example 4.4. Let $n=4, \lambda=(2,2,1,1), L_{1}^{(1)}=6$ and all other $L_{i}^{(a)}=0$. Then

$$
(\nu, J)=\frac{\square}{\square} \square_{0}-2 \square \square 0 \quad \square-1
$$

is an extended rigged configuration in $\widetilde{\mathrm{RC}}(L, \lambda)$, where we have written the parts of $J^{(a, i)}$ next to the parts of length $i$ in partition $\nu^{(a)}$. To see that the riggings form quasipartitions, let us write the vacancy numbers $p_{i}^{(a)}$ next to the parts of length $i$ in partition $\nu^{(a)}$ :

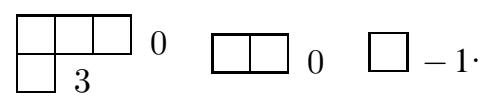

This shows that the labels are indeed all weakly below the vacancy numbers. For

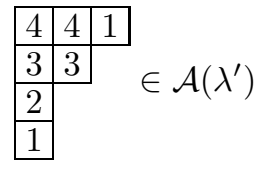

we get the lower bounds

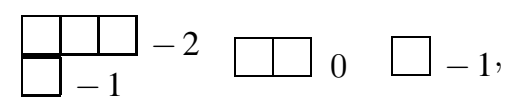

which are less or equal to the riggings in $(\nu, J)$.

\section{Remark 4.5.}


(1) Note that Definition 4.3 is similar to the definition of level-restricted rigged configurations [38, Definition 5.5]. Whereas for level-restricted rigged configurations the vacancy number had to be modified according to tableaux in a certain set, here the lower bounds are modified.

(2) For type $A_{1}$ we have $\lambda=\left(\lambda_{1}, \lambda_{2}\right)$ so that $\mathcal{A}=\{t\}$ contains just the single tableau

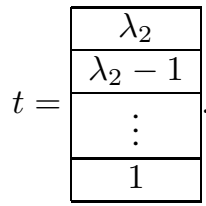

In this case $M_{i}(t)=-\sum_{j=1}^{\lambda_{2}} \chi\left(i \geq t_{j, 1}\right)=-i$. This agrees with the findings of [42].

The next theorem shows that the set of unrestricted rigged configurations $\operatorname{RC}(L, \lambda)$ of type $A_{n-1}^{(1)}$ defined in terms of the crystal structure in Section 3.2 is equal to the set of extended rigged configurations $\widetilde{\mathrm{RC}}(L, \lambda)$ of Definition 4.3.

Theorem 4.6. We have $\mathrm{RC}(L, \lambda)=\widetilde{\mathrm{RC}}(L, \lambda)$.

Proof. Denote by $X_{(\bar{\nu}, \bar{J})}$ the graph with maximal element $(\bar{\nu}, \bar{J}) \in \overline{\mathrm{RC}}(L)$ generated by $f_{a}, e_{a}$ for $a \in \bar{I}$. By definition

$$
\bigcup_{(\bar{\nu}, \bar{J}) \in \overline{\mathrm{RC}}(L)} X_{(\bar{\nu}, \bar{J})}=\mathrm{RC}(L) .
$$

We claim that $\mathrm{RC}(L)=\widetilde{\mathrm{RC}}(L)$. The statement $\mathrm{RC}(L, \lambda)=\widetilde{\mathrm{RC}}(L, \lambda)$ then follows since the weight function is defined in the same way on both sets.

Let $(\bar{\nu}, \bar{J}) \in \overline{\operatorname{RC}}(L, \lambda)$. Then $(\bar{\nu}, \bar{J})$ is admissible with respect to $t \in \mathcal{A}\left(\lambda^{\prime}\right)$ where column $k$ of $t$ is filled with the letters $1,2, \ldots, c_{k}$. Hence $(\bar{\nu}, \bar{J}) \in \widetilde{\mathrm{RC}}(L)$. Now suppose by induction that $(\nu, J) \in \bigcup_{(\bar{\nu}, \bar{J}) \in \overline{\mathrm{RC}}(L)} X_{(\bar{\nu}, \bar{J})}$ is admissible with respect to $t \in \mathcal{A}\left(\lambda^{\prime}\right)$. We claim that $\left(\nu^{\prime}, J^{\prime}\right)=f_{a}(\nu, J)$ is admissible with respect to some $t^{\prime} \in \mathcal{A}\left(\lambda^{\prime \prime}\right)$ where $\lambda^{\prime \prime}$ is obtained from $\lambda^{\prime}$ by adding a box to column $a$. (Note that $f_{a}(\nu, J)=0$ if $\lambda_{a}=0$ or equivalently $c_{a-1}=c_{a}$ ). Let $k$ be the length of the string in $(\nu, J)^{(a)}$ selected by $f_{a}$ (see Definition 3.3). Let $r>k$ be minimal such that $r \notin t_{\text {., } a}$, where $t_{,, a}$ denotes column $a$ of $t$. Similarly, let $s>k$ be minimal such that $s \in t_{\cdot, a+1}$. Then $t^{\prime}$ is obtained from $t$ by adding $r$ to column $a$, and by removing $s$ from column $a+1$ and adding $c_{a}+1$ to column $a+1$ in such a way that the columns are still strictly decreasing. Note that $t^{\prime}$ is by construction strictly decreasing in columns and has the property that the elements in column $b$ lie in the set $\left\{1,2, \ldots, c_{b-1}^{\prime}\right\}$ where $c_{b}^{\prime}=c_{b}+\delta_{a, b}$ is the length of column $b$ in $t^{\prime}$. Hence $t^{\prime} \in \mathcal{A}\left(\lambda^{\prime \prime}\right)$.

To see that $\left(\nu^{\prime}, J^{\prime}\right)=f_{a}(\nu, J)$ is admissible with respect to $t^{\prime}$, note that strings in $(\nu, J)^{(a-1)}$ and $(\nu, J)^{(a+1)}$ change by $(j, x) \mapsto(j, x+\chi(j>k))$, and strings in $(\nu, J)^{(a)}$ change by $(j, x) \mapsto(j, x-2 \chi(j>k))$. In addition to this there is a new string $(k+$ $1,-m-1)$ in $\left(\nu^{\prime}, J^{\prime}\right)^{(a)}$ where $-m$ is the smallest label in $(\nu, J)^{(a)}$. Since column $a$ of $t^{\prime}$ contains an additional entry greater than $k$ and in column $a+1$ an entry greater than $k$ was increased, $t^{\prime}$ certainly provides valid lower bounds for $\left(\nu^{\prime}, J^{\prime}\right)^{(a \pm 1)}$. Note that

$$
M_{j+1}^{(a)}(t)=M_{j}^{(a)}(t)-\chi\left(j+1 \in t_{\cdot, a}\right)+\chi\left(j+1 \in t_{\cdot, a+1}\right) .
$$


Since by definition of $f_{a}, k$ is largest such that there is a string of this length with label $-m$, it is not hard to check that $t^{\prime}$ gives proper lower bounds for $\left(\nu^{\prime}, J^{\prime}\right)$. This shows that

$$
\bigcup_{(\bar{\nu}, \bar{J}) \in \overline{\mathrm{RC}}(L)} X_{(\bar{\nu}, \bar{J})} \subset \widetilde{\mathrm{RC}}(L) .
$$

To prove the reverse inclusion, suppose $(\nu, J) \in \widetilde{\mathrm{RC}}(L)$. Let $t \in \mathcal{A}\left(\lambda^{\prime}\right)$ be such that $(\nu, J)$ is admissible with respect to $t$. If $(\nu, J) \in \overline{\mathrm{RC}}(L)$, then certainly $(\nu, J) \in$ $\bigcup_{(\bar{\nu}, \bar{J}) \in \overline{\mathrm{RC}}(L)} X_{(\bar{\nu}, \bar{J})}$. If $(\nu, J) \notin \overline{\mathrm{RC}}(L)$, there must be at least one negative rigging. Suppose this occurs in $(\nu, J)^{(a)}$. Then $e_{a}(\nu, J)$ exists. To see this note that all colabels remain fixed, so that all labels are still weakly below the vacancy number. The string $(k,-m)$ in $(\nu, J)^{(a)}$ selected by $e_{a}$ becomes $(k-1,-m+1)$. Since by the definition of $k, k$ is smallest such that its label is $-m<0$, all labels of strings of length less than $k$ are strictly bigger than $-m$. Hence $p_{j}^{(a)}>-m$ for all $j<k$ such that $j$ appears as a part. By the convexity property (3.4) of $p_{j}^{(a)}$, this is true for all $j<k$. Hence $e_{a}(\nu, J)$ exists.

Next we need to show that $\left(\nu^{\prime}, J^{\prime}\right)=e_{a}(\nu, J) \in \widetilde{\mathrm{RC}}(L)$. Let $r \leq k$ be maximal such that $r \in t_{,, a}$ and let $s \leq k$ be maximal such that $s \notin t_{, a+1}$. Note that $r$ and $s$ must exist, since the rigging of the string of length $k$ in $(\nu, J)^{(a)}$ is negative so that $M_{k}^{(a)}(t)<0$. But this implies that $\#\left\{j \in t_{,, a} \mid j \leq k\right\}>\#\left\{j \in t_{\text {., } a+1} \mid j \leq k\right\}$. Then define $t^{\prime}$ by removing $r$ from column $a$ of $t$ and changing the largest element in column $a+1$ to $s$. By similar arguments as for the previous case, $e_{a}(\nu, J)$ is admissible with respect to $t^{\prime}$.

Remark 4.7. For type $D_{n}^{(1)}$, a simple characterization in terms of lower bounds for the parts of a configuration $\nu \in C(L)$ does not seem to exist. For example take $B=B^{2,1}$ of type $D_{4}^{(1)}$ so that $L_{1}^{(2)}=1$ and all other $L_{i}^{(a)}=0$. Then the unrestricted rigged configurations

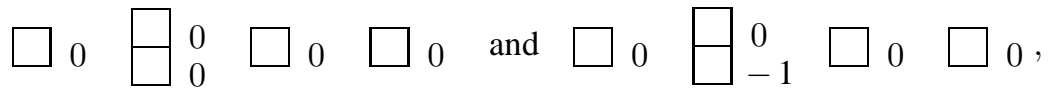

which correspond to the crystal elements $\frac{1}{\overline{1}}$ and $\frac{3}{\overline{3}}$ respectively, occur in $\operatorname{RC}(L)$, but

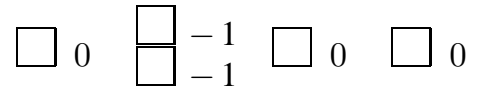

on the other hand does not appear.

4.2. Fermionic formula. With the explicit characterization of the unrestricted rigged configurations of Section 4.1, it is possible to derive an explicit formula for the polynomials $M(L, \lambda)$ of (3.12).

Let $\mathcal{S} \mathcal{A}\left(\lambda^{\prime}\right)$ be the set of all nonempty subsets of $\mathcal{A}\left(\lambda^{\prime}\right)$ and set

$$
M_{i}^{(a)}(S)=\max \left\{M_{i}^{(a)}(t) \mid t \in S\right\} \quad \text { for } S \in \mathcal{S} \mathcal{A}\left(\lambda^{\prime}\right) .
$$

By inclusion-exclusion the set of all allowed riggings for a given $\nu \in \mathrm{C}(L, \lambda)$ is

$$
\bigcup_{S \in \mathcal{S} \mathcal{A}\left(\lambda^{\prime}\right)}(-1)^{|S|+1}\left\{J \mid J^{(a, i)} \text { is a }\left(M_{i}^{(a)}(S), p_{i}^{(a)}, m_{i}^{(a)}\right) \text {-quasipartition }\right\} .
$$

The $q$-binomial coefficient $\left[\begin{array}{c}m+p \\ m\end{array}\right]$, defined as

$$
\left[\begin{array}{c}
m+p \\
m
\end{array}\right]=\frac{(q)_{m+p}}{(q)_{m}(q)_{p}}
$$


where $(q)_{n}=(1-q)\left(1-q^{2}\right) \cdots\left(1-q^{n}\right)$, is the generating function of partitions with at most $m$ parts each not exceeding $p$. Hence the polynomial $M(L, \lambda)$ may be rewritten as

$$
\begin{aligned}
M(L, \lambda)=\sum_{S \in \mathcal{S} \mathcal{A}\left(\lambda^{\prime}\right)}(-1)^{|S|+1} \sum_{\nu \in \mathrm{C}(L, \lambda)} q^{c c(\nu)+\sum_{(a, i) \in \mathcal{H}} m_{i}^{(a)} M_{i}^{(a)}(S)} & \\
& \times \prod_{(a, i) \in \mathcal{H}}\left[\begin{array}{c}
m_{i}^{(a)}+p_{i}^{(a)}-M_{i}^{(a)}(S) \\
m_{i}^{(a)}
\end{array}\right]
\end{aligned}
$$

called fermionic formula. By Corollary 3.11 this is also a formula for the unrestricted configuration sum $X(B, \lambda)$. As mentioned in the introduction, this formula is different from the fermionic formulas of $[15,20]$ which exist in the special case when $L$ is the multiplicity array of $B=B^{1, s_{k}} \otimes \cdots \otimes B^{1, s_{1}}$ or $B=B^{r_{k}, 1} \otimes \cdots \otimes B^{r_{1}, 1}$.

4.3. The Kashiwara operators $e_{0}$ and $f_{0}$. The Kirillov-Reshetikhin crystals $B^{r, s}$ are affine crystals and admit the Kashiwara operators $e_{0}$ and $f_{0}$. It was shown in [40] that for type $A_{n-1}^{(1)}$ they can be defined in terms of the promotion operator pr as

$$
e_{0}=\mathrm{pr}^{-1} \circ e_{1} \circ \mathrm{pr} \quad \text { and } f_{0}=\mathrm{pr}^{-1} \circ f_{1} \circ \mathrm{pr} \text {. }
$$

The promotion operator is a bijection pr $: B \rightarrow B$ such that the following diagram commutes for all $a \in I$

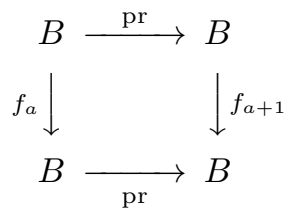

and such that for every $b \in B$ the weight is rotated

$$
\left\langle h_{a+1}, \operatorname{wt}(\operatorname{pr}(b))\right\rangle=\left\langle h_{a}, \operatorname{wt}(b)\right\rangle .
$$

Here subscripts are taken modulo $n$.

We are now going to define the promotion operator on unrestricted rigged configurations.

Definition 4.8. Let $(\nu, J) \in \mathrm{RC}(L, \lambda)$. Then $\operatorname{pr}(\nu, J)$ is obtained as follows:

(1) Set $\left(\nu^{\prime}, J^{\prime}\right)=f_{1}^{\lambda_{1}} f_{2}^{\lambda_{2}} \cdots f_{n}^{\lambda_{n}}(\nu, J)$ where $f_{n}$ acts on $(\nu, J)^{(n)}=\emptyset$.

(2) Apply the following algorithm $\rho$ to $\left(\nu^{\prime}, J^{\prime}\right) \lambda_{n}$ times: Find the smallest singular string in $\left(\nu^{\prime}, J^{\prime}\right)^{(n)}$. Let the length be $\ell^{(n)}$. Repeatedly find the smallest singular string in $\left(\nu^{\prime}, J^{\prime}\right)^{(k)}$ of length $\ell^{(k)} \geq \ell^{(k+1)}$ for all $1 \leq k<n$. Shorten the selected strings by one and make them singular again.

Example 4.9. Let $B=B^{2,2}, L$ the corresponding multiplicity array and $\lambda=(1,0,1,2)$. Then

$$
(\nu, J)=\square 0 \square_{-1}^{-1} \square_{-1} \in \mathrm{RC}(L, \lambda)
$$

corresponds to the tableau $b=$\begin{tabular}{|l|l|}
\hline 1 & 3 \\
\hline 4 & 4 \\
\hline
\end{tabular}$\in \mathcal{P}(B, \lambda)$. After step (1) of Definition 4.8 we have

$$
\left(\nu^{\prime}, J^{\prime}\right)=\square \square-1 \square_{0} \square_{-1}^{\square} \square_{-1} \square_{-1}^{\square}
$$


Then applying step (2) yields

$$
\operatorname{pr}(\nu, J)=\emptyset \square 0 \quad \square-1
$$

which corresponds to the tableau $\operatorname{pr}(b)=$\begin{tabular}{|l|l|}
1 & 1 \\
\hline 2 & 4 \\
\hline
\end{tabular} .

Lemma 4.10. The map pr of Definition 4.8 is well-defined and satisfies (4.3) for $1 \leq a \leq$ $n-2$ and (4.4) for $0 \leq a \leq n-1$.

Proof. To prove that pr is well-defined we need to show that singular strings of length $\ell^{(k)}$ exist in $\left(\nu^{\prime}, J^{\prime}\right)^{(k)}=f_{1}^{\lambda_{1}} f_{2}^{\lambda_{2}} \cdots f_{n}^{\lambda_{n}}(\nu, J)$, where $\ell^{(1)} \geq \ell^{(2)} \geq \cdots \geq \ell^{(n)}$. For a given $1 \leq a \leq n$, set $(\tilde{\nu}, \tilde{J})=f_{a}^{\lambda_{a}} f_{a+1}^{\lambda_{a}+1} \cdots f_{n}^{\lambda_{n}}(\nu, J)$. By definition $\varphi_{a}(\tilde{\nu}, \tilde{J})=0$. Hence by Lemma $3.6 p=s$ where $p=p_{i}^{(a)}$ for large $i$ and $s$ is the smallest nonpositive label in $(\tilde{\nu}, \tilde{J})^{(a)}$. Let $\ell$ be the length of the largest part in $(\tilde{\nu}, \tilde{J})^{(a)}$. Suppose that $(\tilde{\nu}, \tilde{J})^{(a-1)}$ or $(\tilde{\nu}, \tilde{J})^{(a+1)}$ has a part of length bigger than $\ell$. Then by the definition of the vacancy number, $p_{\ell}^{(a)}<p$. But this contradicts the fact that $s=p$ is the smallest label in $(\tilde{\nu}, \tilde{J})^{(a)}$. Hence the parts of $(\tilde{\nu}, \tilde{J})^{(a \pm 1)}$ cannot exceed $\ell$ and the string $(\ell, p)$ in $(\tilde{\nu}, \tilde{J})^{(a)}$ is singular. Since the application of $f_{1}^{\lambda_{1}} \cdots f_{a-1}^{\lambda_{a-1}}$ does not change the colabels in the $a$-th rigged partition, the largest string remains singular. Note that the above argument also shows that the longest parts in $\nu^{\prime(a)}$ decrease with $a$. Hence there exist singular strings in $\left(\nu^{\prime}, J^{\prime}\right)$ such that $\ell^{(k)} \geq \ell^{(k+1)}$ and pr is well-defined.

Next we show that pr satisfies (4.4). Let $(\nu, J) \in \operatorname{RC}(L, \lambda)$ so that $\operatorname{wt}(\nu, J)=\lambda=$ $\left(\lambda_{1}, \ldots, \lambda_{n}\right)$. After step (1) of Definition 4.8, we have $\left|\nu^{(a)}\right| \mapsto\left|\nu^{(a)}\right|+\lambda_{a}$. Hence by (3.8), noting that $\sum_{i} i m_{i}^{(a)}=\left|\nu^{(a)}\right|$, we obtain

$$
\begin{aligned}
\operatorname{wt}\left(\nu^{\prime}, J^{\prime}\right) & =\operatorname{wt}(\nu, J)-\sum_{a \in \bar{I}} \lambda_{a} \alpha_{a} \\
& =\left(\lambda_{1}, \ldots, \lambda_{n}, 0\right)+\left(-\lambda_{1}, \lambda_{1}-\lambda_{2}, \ldots, \lambda_{n-1}-\lambda_{n}, \lambda_{n}\right)=\left(0, \lambda_{1}, \ldots, \lambda_{n}\right)
\end{aligned}
$$

where in the second line we added an $(n+1)$-th component to the weight. In step (2) of Definition 4.8 the size of the $a$-th partition changes as $\left|\nu^{(a)}\right| \mapsto\left|\nu^{(a)}\right|-\lambda_{n}$. Hence

$$
\begin{aligned}
\operatorname{wt}(\operatorname{pr}(\nu, J)) & =\operatorname{wt}\left(\nu^{\prime}, J^{\prime}\right)-\sum_{a \in \bar{I}} \lambda_{n} \alpha_{a} \\
& =\left(0, \lambda_{1}, \ldots, \lambda_{n}\right)-\left(-\lambda_{n}, 0, \ldots, 0, \lambda_{n}\right)=\left(\lambda_{n}, \lambda_{1}, \lambda_{2}, \ldots, \lambda_{n-1}, 0\right) .
\end{aligned}
$$

Dropping the last component (which we only added for the intermediate calculation) we obtain (4.4).

It remains to prove (4.3). We treat step (1) and step (2) in the Definition 4.8 of pr separately. Note that $f_{a}$ and $f_{b}$ commute as long as $b \neq a \pm 1$. Hence for step (1) it suffices to show that for $(\nu, J) \in \operatorname{RC}(L, \lambda)$ with $\lambda_{a+2}=0$ we have $f_{a+1} f_{a}^{\lambda_{a}} f_{a+1}^{\lambda_{a+1}}(\nu, J)=$ $f_{a}^{\lambda_{a}-1} f_{a+1}^{\lambda_{a+1}+1} f_{a}(\nu, J)$. Note that it is not hard to check that Lemma 3.6 implies

$$
\nabla_{a} \varphi_{a+1}(\nu, J)= \begin{cases}0 & \text { if } k_{a}^{f} \leq k_{a+1}^{e}, \\ -1 & \text { if } k_{a}^{f}>k_{a+1}^{e},\end{cases}
$$

where $k_{a}^{f}$ (resp. $\left.k_{a+1}^{e}\right)$ is the length of the string in $(\nu, J)^{(a)}$ (resp. $\left.(\nu, J)^{(a+1)}\right)$ selected by $f_{a}$ (resp. $\left.e_{a+1}\right)$. Since in our case $\nabla_{a} \varphi_{a+1}(\nu, J)=-1$ we must have $k_{a}^{f}>k_{a+1}^{e}$. Let $\ell_{a}^{f}$ be the length of the string selected by $f_{a}$ in $f_{a+1}^{\lambda_{a+1}}(\nu, J)$. If $\ell_{a}^{f}>k_{a+1}^{f}$, then necessarily $k_{a}^{f}=\ell_{a}^{f}$ since the application of $f_{a+1}$ only increases labels in the $a$-th rigged partition. 
Note that in this case $f_{a} f_{a+1}(\nu, J)=f_{a+1} f_{a}(\nu, J)$. Hence it suffices to repeat the analysis for $f_{a} f_{a+1}(\nu, J)$. If $\ell_{a}^{f} \leq k_{a+1}^{f}$, it can be checked explicitly that $f_{a+1} f_{a}^{\lambda_{a}} f_{a+1}^{\lambda_{a+1}}(\nu, J)=$ $f_{a}^{\lambda_{a}-1} f_{a+1}^{\lambda_{a+1}+1} f_{a}(\nu, J)$.

The algorithm $\rho$ in step (2) of Definition 4.8 commutes with $f_{a}$ for all $2 \leq a \leq n-1$ (assuming that both $\rho$ and $f_{a}$ are defined on the rigged configuration)

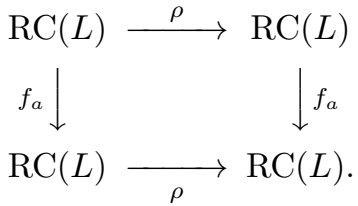

To see this, let $(\nu, J) \in \operatorname{RC}(L)$, let $(k, s)$ be the string of length $k$ and label $s$ selected by $f_{a}$ in $(\nu, J)$ and denote by $\ell^{(b)}$ the length of the strings selected by $\rho$ in $(\nu, J)$. If $k>\ell^{(a)}$ or $k<\ell^{(a+1)}-1$, then $\rho$ and $f_{a}$ obviously commute since $f_{a}$ leaves all colabels of unselected strings fixed and $\rho$ leaves all labels of unselected strings fixed. Hence assume that $\ell^{(a+1)}-1 \leq k \leq \ell^{(a)}$. Since $s$ is the smallest label occurring in $(\nu, J)^{(a)}$, we must have $p_{i}^{(a)} \geq s$ for all $i$ such that $m_{i}^{(a)}>0$. Because of the convexity property (3.4) of the vacancy numbers, it follows that $p_{i}^{(a)} \geq s$ for all $i$. Since $(k, s)$ is the largest string in $(\nu, J)^{(a)}$ with label $s$, all strings $(i, x)$ with $i>k$ in $(\nu, J)^{(a)}$ satisfy $x>s$. This together with the convexity condition (3.4) implies that $p_{i}^{(a)} \geq s+1$ for all $i>k$. Also, since there are no singular strings of length $k<i<\ell^{(a)}$ in $(\nu, J)^{(a)}$, we must have $p_{i}^{(a)} \geq s+2$ for $k<i<\ell^{(a)}$ and $m_{i}^{(a)}>0$.

Let us first assume that $k=\ell^{(a)}$. Then either $m_{k}^{(a)} \geq 2$, or $m_{k}^{(a)}=1$ and $p_{k}^{(a)}=s$. First consider the case that $m_{k}^{(a)}=1$ and $p_{k}^{(a)}=s$. For $i=k$ the inequality (3.3) reads

$$
2 \geq m_{k}^{(a-1)}+m_{k}^{(a+1)}+\left(p_{k-1}^{(a)}-p_{k}^{(a)}\right)+\left(p_{k+1}^{(a)}-p_{k}^{(a)}\right) .
$$

Certainly $p_{k+1}^{(a)}>p_{k}^{(a)}=s$ as discussed above. Similarly, $p_{k-1}^{(a)} \geq p_{k}^{(a)}$ since $s$ is the smallest label in $(\nu, J)^{(a)}$. This implies that $p_{k-1}^{(a)}=p_{k}^{(a)}$ or $p_{k-1}^{(a)}=p_{k}^{(a)}+1$. If $\ell^{(a+1)}<$ $\ell^{(a)}$ we must have $p_{k-1}^{(a)}=p_{k}^{(a)}+1$. This is clear if $m_{k-1}^{(a)}>0$ since else $\rho$ would pick the singular string of length $k-1$ rather than $k$. If $m_{k-1}^{(a)}=0$ let $k^{\prime}<k$ be maximal such that $m_{k^{\prime}}^{(a)}>0$. By (3.3) it follows that $m_{i}^{(a+1)}=0$ for $k^{\prime}<i<k$ and $p_{i}^{(a)}=s$ for $k^{\prime} \leq i \leq k$ if $p_{k}^{(a)}=p_{k-1}^{(a)}=s$. But then $\ell^{(a+1)} \leq k^{\prime}$ and $\rho$ would pick the singular string of length $k^{\prime}$ in $(\nu, J)^{(a)}$ which is a contradiction to our assumption $\ell^{(a)}=k$. Hence $p_{k-1}^{(a)}=p_{k}^{(a)}+1$ if $\ell^{(a+1)}<\ell^{(a)}$. If $\ell^{(a+1)}=\ell^{(a)}=k$ we must have $m_{k}^{(a+1)}>0$ so that $p_{k-1}^{(a)}=p_{k}^{(a)}$. Now after the application of $\rho$ there is a new string $\left(k-1, p_{k-1}^{(a)}-\chi\left(\ell^{(a+1)}<\ell^{(a)}\right)\right)=(k-1, s)$. Since this is the longest string with label $s$, it will be picked by $f_{a}$ to give the string $(k, s-1)$. If $f_{a}$ is applied first, there is a new string $(k+1, s-1)$. By (4.6) and the previous findings, we have $p_{k+1}^{(a)}=s+1$. Under $f_{a}$ the vacancy number changes to $p_{k+1}^{(a)} \mapsto p_{k+1}^{(a)}-2=s-1$. Hence the string $(k+1, s-1)$ in $f_{a}(\nu, J)^{(a)}$ is singular and will be picked by $\rho$. Note also that by (4.6) $m_{k}^{(a-1)}=0$ so that $\ell^{(a-1)}>k=\ell^{(a)}$. This shows that $f_{a}$ and $\rho$ commute in this case.

If $k=\ell^{(a)}$ and $m_{k}^{(a)} \geq 2$ it needs to be shown that the string of length $k$ picked by $f_{a}$ is still picked after the application of $\rho$. The only case in which this might not happen is when the new string $(k-1, x)$ produced by $\rho$ has label $x<s$. Note that $p_{k-1}^{(a)} \geq s$ 
and $p_{k-1}^{(a)} \mapsto p_{k-1}^{(a)}-\chi\left(\ell^{(a+1)}<k\right)=x$ under $\rho$. Hence there is only a problem if $p_{k-1}^{(a)}=p_{k}^{(a)}=s$ and $\ell^{(a+1)}<k$. Distinguishing the two cases $m_{k-1}^{(a)}>0$ and $m_{k-1}^{(a)}=0$, by very similar arguments as above this is not possible. Therefore $\rho$ and $f_{a}$ commute.

Hence from now on we assume that $k<\ell^{(a)}=: \ell$. Note that $p_{\ell}^{(a)} \geq s+1$ since all strings of length greater than $k$ have label greater than $s$. Also $p_{k}^{(a)} \geq s+1$ if $k \geq \ell^{(a+1)}$ since in this case the string $(k, s)$ cannot be singular. Convexity (3.4) implies that $p_{\ell-1}^{(a)} \geq$ $s+2$ and $p_{k+1}^{(a)} \geq s+2$ unless $m_{i}^{(a)}=0$ for all $k<i<\ell$ and $p_{i}^{(a)}=s+1$ for all $k \leq i \leq \ell$, or $k=\ell^{(a+1)}-1$. If $p_{\ell-1}^{(a)} \geq s+2$ and $p_{k+1}^{(a)} \geq s+2, f_{a}$ creates a new string $(k+1, s-1)$ with new vacancy number $p_{k+1}^{(a)} \geq s$. Hence this string is not singular and $\rho$ still picks the string $\left(\ell, p_{\ell}^{(a)}\right)$. Applying $\rho$ first creates a new string $\left(\ell-1, p_{\ell-1}^{(a)}-1\right)$ with label $p_{\ell-1}^{(a)}-1 \geq s+1$. Hence $f_{a}$ picks the same string $(k, s)$ as before which shows that $f_{a}$ and $\rho$ commute.

Now assume that $m_{i}^{(a)}=0$ for $k<i<\ell, p_{i}^{(a)}=s+1$ for $k \leq i \leq \ell$ and $k \geq \ell^{(a+1)}$. In this case $f_{a}$ makes a new string $(k+1, s-1)$ which is singular since $p_{k+1}^{(a)} \mapsto p_{k+1}^{(a)}-2=$ $s-1$. Then $\rho$ picks this string and makes it into $(k, s)$ since under $\rho \circ f_{a}$ the vacancy number changes to $p_{k}^{(a)} \mapsto p_{k}^{(a)}-1=s$. On the other hand, first applying $\rho$ picks the string $(\ell, s+1)$ and makes it into $(\ell-1, s)$. Since this is now the largest string with label $s, f_{a}$ picks it and makes it into $(\ell, s-1)$ which is the same as under $\rho \circ f_{a}$ since recall that $f_{a}$ does not change colabels but $p_{\ell}^{(a)} \mapsto p_{\ell}^{(a)}-2=s-1$ under $f_{a}$. Since $m_{i}^{(a-1)}=0$ for all $k<i<\ell$ by (3.3), $\ell^{(a-1)}$ remains unchanged. Hence $f_{a}$ and $\rho$ commute.

Finally assume that $k=\ell^{(a+1)}-1$ and $p_{k+1}^{(a)}=s+1$. This implies that $m_{i}^{(a)}=0$ for $k<i<\ell$. By (3.3) with $i=k+1$ and $\ell>k+1$ we obtain

$$
0 \geq m_{k+1}^{(a-1)}+m_{k+1}^{(a+1)}+\left(p_{k}^{(a)}-p_{k+1}^{(a)}\right)+\left(p_{k+2}^{(a)}-p_{k+1}^{(a)}\right) .
$$

Since $\ell^{(a+1)}=k+1$ we have $m_{k+1}^{(a+1)} \geq 1$. Also $p_{k}^{(a)}-p_{k+1}^{(a)}=p_{k}^{(a)}-s-1 \geq-1$ and $p_{k+2}^{(a)}-p_{k+1}^{(a)} \geq 0$ since $p_{k}^{(a)} \geq s$ and $p_{k+2}^{(a)} \geq s+1$. Hence we must have $p_{k}^{(a)}=s$. Now $f_{a}$ creates a new string $(k+1, s-1)$ which is singular since $p_{k+1}^{(a)} \mapsto p_{k+1}^{(a)}-2=s-1$. Then $\rho$ picks this string and makes a string $(k, s)$. If $\rho$ is applied first it transforms the string $(\ell, s+1)$ to $(\ell-1, s)$. Then this becomes the longest string with label $s$, so that $f_{a}$ picks it and transforms it into $(\ell, s-1)$. Hence $f_{a}$ and $\rho$ commute. If $\ell=k+1$ it is easy to show that $f_{a}$ and $\rho$ commute.

This concludes the proof of Lemma 4.10.

Lemma 7 of [40] states that for a single Kirillov-Reshetikhin crystal $B=B^{r, s}$ the promotion operator pr is uniquely determined by (4.3) for $1 \leq a \leq n-2$ and (4.4) for $0 \leq a \leq n-1$. Hence by Lemma $4.10 \mathrm{pr}$ on $\mathrm{RC}(L)$ is indeed the correct promotion operator when $L$ is the multiplicity array of $B=B^{r, s}$.

Theorem 4.11. Let $L$ be the multiplicity array of $B=B^{r, s}$. Then $\operatorname{pr}: \operatorname{RC}(L) \rightarrow \operatorname{RC}(L)$ of Definition 4.8 is the promotion operator on rigged configurations.

Conjecture 4.12. Theorem 4.11 is true for any $B=B^{r_{k}, s_{k}} \otimes \cdots \otimes B^{r_{1}, s_{1}}$.

Unfortunately, the characterization [40, Lemma 7] does not suffice to define pr uniquely on tensor products $B=B^{r_{k}, s_{k}} \otimes \cdots \otimes B^{r_{1}, s_{1}}$. In [10] a bijection $\Phi: \mathcal{P}(B, \lambda) \rightarrow \operatorname{RC}(L, \lambda)$ is defined via a direct algorithm. It is expected that Conjecture 4.12 can be proven by 
showing that the following diagram commutes:

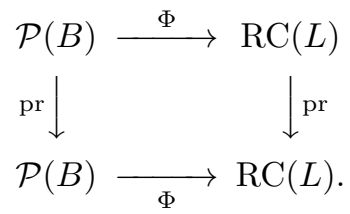

Alternatively, an independent characterization of pr on tensor factors would give a new, more conceptual way of defining the bijection $\Phi$ between paths and (unrestricted) rigged configurations. A proof that the crystal operators $f_{a}$ and $e_{a}$ commute with $\Phi$ for $a=$ $1,2, \ldots, n-1$ is given in [10].

\section{REFERENCES}

[1] G. E. Andrews, Multiple series Rogers-Ramanujan type identities, Pacific J. Math. 114 (1984) 267-283.

[2] G.E. Andrews, A. Schilling, S.O. Warnaar, An $A_{2}$ Bailey lemma and Rogers-Ramanujan-type identities, J. Amer. Math. Soc. 12 (1999), no. 3, 677-702.

[3] G. Albertini, S. Dasmahapatra, B. M. McCoy, Spectrum and completeness of the integrable 3-state Potts model: a finite size study, Adv. Ser. Math. Phys. 16 (1992) 1-53.

[4] W. N. Bailey, Identities of the Rogers-Ramanujan type, Proc. London Math. Soc. (2) 50 (1949) 1-10.

[5] R. J. Baxter, Exactly solved models in statistical mechanics, Academic Press, London, 1982.

[6] H. A. Bethe, Zur Theorie der Metalle, I. Eigenwerte und Eigenfunktionen der linearen Atomkette, Z. Physik 71 (1931) 205-231.

[7] V. Chari, A. Pressley, Quantum affine algebras and their representations, Representations of groups (Banff, AB, 1994), 59-78, CMS Conf. Proc., 16, Amer. Math. Soc., Providence, RI, 1995.

[8] V. Chari, A. Pressley, Twisted quantum affine algebras, Comm. Math. Phys. 196 (1998) 461-476.

[9] L. Deka, A. Schilling, New explicit expression for $A_{n}^{(1)}$ supernomials, Extended Abstract, 17th International Conference on Formal Power Series and Algebraic Combinatorics 2005, University of Messina, Italy, June 2005.

[10] L. Deka, A. Schilling, New fermionic formula for unrestricted Kostka polynomials, in preparation.

[11] J. Haglund, M. Haiman, N. Loehr, A combinatorial formula for Macdonald polynomials, J. Amer. Math. Soc. 18 (2005), no. 3, 735-761.

[12] K. Hikami, R. Inoue, Y. Komori, Crystallization of the Bogoyavlensky lattice, J. Phys. Soc. Japan 68 (1999), no. 7, 2234-2240.

[13] G. Hatayama, A. Kuniba, T. Takagi, Soliton cellular automata associated with crystal bases, Nuclear Phys. B 577 (2000), no. 3, 619-645.

[14] G. Hatayama, K. Hikami, R. Inoue, A. Kuniba, T. Takagi, T. Tokihiro, The $A_{M}^{(1)}$ automata related to crystals of symmetric tensors, J. Math. Phys. 42 (2001), no. 1, 274-308.

[15] G. Hatayama, A.N. Kirillov, A. Kuniba, M. Okado, T. Takagi, Y. Yamada, Character formulae of $\widehat{\mathrm{sl}}_{n}$ modules and inhomogeneous paths, Nuclear Phys. B 536 (1999), no. 3, 575-616.

[16] G. Hatayama, A. Kuniba, M. Okado, T. Takagi, Z. Tsuboi, Paths, crystals and fermionic formulae, MathPhys odyssey, 2001, 205-272, Prog. Math. Phys., 23, Birkhäuser Boston, Boston, MA, 2002.

[17] G. Hatayama, A. Kuniba, M. Okado, T. Takagi, Y. Yamada, Remarks on fermionic formula, Contemp. Math. 248 (1999) 243-291.

[18] M. Kashiwara, Crystalizing the q-analogue of universal enveloping algebras, Comm. Math. Phys. 133 (1990), no. 2, 249-260.

[19] M. Kashiwara, On crystal bases, Representations of groups (Banff, AB, 1994), 155-197, CMS Conf. Proc., 16, Amer. Math. Soc., Providence, RI, 1995.

[20] A.N. Kirillov, New combinatorial formula for modified Hall-Littlewood polynomials, Contemp. Math. 254 (2000) 283-333.

[21] Y. Koga, Level one perfect crystals for $B_{n}^{(1)}, C_{n}^{(1)}$ and $D_{n}^{(1)}$, J. Alg. 217 (1999) 312-334.

[22] R. Kedem, T.R. Klassen, B.M. McCoy, E. Melzer, Fermionic quasi-particle representations for characters of $\left(G^{(1)}\right)_{1} \times\left(G^{(1)}\right)_{1} /\left(G^{(1)}\right)_{2}$, Phys. Lett. B 304 (1993), no. 3-4, 263-270.

[23] R. Kedem, T.R. Klassen, B.M. McCoy, E. Melzer, Fermionic sum representations for conformal field theory characters, Phys. Lett. B 307 (1993), no. 1-2, 68-76. 
[24] S.-J. Kang, M. Kashiwara, K. C. Misra, Crystal bases of Verma modules for quantum affine Lie algebras, Compositio Math. 92 (1994) 299-325.

[25] S.-J. Kang, M. Kashiwara, K.C. Misra, T. Miwa, T. Nakashima, A. Nakayashiki, Perfect crystals of quantum affine Lie algebras, Duke Math. J. 68 (1992) 499-607.

[26] A. Kuniba, M. Okado, R. Sakamoto, T. Takagi, Y. Yamada, private communication.

[27] S. V. Kerov, A. N. Kirillov, N. Y. Reshetikhin, Combinatorics, the Bethe ansatz and representations of the symmetric group J. Soviet Math. 41 (1988), no. 2, 916-924.

[28] A. N. Kirillov, N. Y. Reshetikhin, The Bethe Ansatz and the combinatorics of Young tableaux, J. Soviet Math. 41 (1988) 925-955.

[29] A.N. Kirillov, M. Shimozono, A generalization of the Kostka-Foulkes polynomials, J. Algebraic Combin. 15 (2002), no. 1, 27-69.

[30] A. N. Kirillov, A. Schilling, M. Shimozono, A bijection between Littlewood-Richardson tableaux and rigged configurations, Selecta Math. (N.S.) 8 (2002), no. 1, 67-135.

[31] A. Lascoux, B. Leclerc, J.-Y. Thibon, Ribbon tableaux, Hall-Littlewood functions, quantum affine algebras, and unipotent varieties, J. Math. Phys. 38 (1997), no. 2, 1041-1068.

[32] B. Leclerc, J.-Y. Thibon, Littlewood-Richardson coefficients and Kazhdan-Lusztig polynomials, Adv. Stud. Pure Math. 28 (2000) 155-220.

[33] M. Okado, A. Schilling, M. Shimozono, A crystal to rigged configuration bijection for nonexceptional affine algebras, "Algebraic Combinatorics and Quantum Groups", Edited by N. Jing, World Scientific (2003), 85124.

[34] M. Okado, A. Schilling, M. Shimozono, Virtual crystals and fermionic formulas of type $D_{n+1}^{(2)}, A_{2 n}^{(2)}$, and $C_{n}^{(1)}$, Represent. Theory 7 (2003) 101-163.

[35] M. Okado, A. Schilling, M. Shimozono, Virtual crystals and Kleber's algorithm, Comm. Math. Phys. 238 (2003), no. 1-2, 187-209.

[36] A. Schilling, q-supernomial coefficients: from riggings to ribbons, MathPhys odyssey, 2001, 437-454, Prog. Math. Phys., 23, Birkhäuser Boston, Boston, MA, 2002.

[37] A. Schilling, A bijection between type $D_{n}^{(1)}$ crystals and rigged configurations, J. Algebra 285 (2005) 292334.

[38] A. Schilling, M. Shimozono, Fermionic formulas for level-restricted generalized Kostka polynomials and coset branching functions, Commun. Math. Phys. 220 (2001) 105-164.

[39] A. Schilling, M. Shimozono, $X=M$ for symmetric powers, J. Algebra, to appear; math.QA/0412376.

[40] M. Shimozono, Affine type A crystal structure on tensor products of rectangles, Demazure characters, and nilpotent varieties, J. Algebraic Combin. 15 (2002) 151-187.

[41] J. R. Stembridge, A local characterization of simply-laced crystals, Transactions of the AMS 355 (2003) 4807-4823.

[42] T. Takagi, Inverse scattering method for a soliton cellular automaton, Nuclear Phys. B 707 (2005) 577-601.

[43] S.O. Warnaar, The Bailey lemma and Kostka polynomials, J. Algebraic Combin. 20 (2004), no. 2, 131-171.

Department of Mathematics, University of California, One Shields Avenue, Davis, CA 95616-8633, U.S.A.

E-mail address: anne@math.ucdavis.edu

URL: http://www. math.ucdavis.edu/ anne 